\title{
STUDIES ON THE CORNEAL AND TRABECULAR ENDOTHELIUM \\ II. ENDOTHELIUM OF THE ZONE OF TRANSITION*
}

\author{
BY \\ F. VRABEC \\ From the First Eye Clinic, University of Prague, Czechoslovakia
}

AT the periphery of the cornea the corneal endothelium passes over the margin of Descemet's membrane to the trabecular meshwork (Vrabec, 1957). The size and shape of the cells of the corneal endothelium undergo a peculiar change in approaching this region (Vrabec, 1958a). A study by means of the replica method (Vrabec, 1958b) demonstrated that the endothelial cells became elongated in the meridional direction and then lost their outlines in the region of the anterior border of Schwalbe's ring. Only a few nuclei were seen by the replica technique.

The results of examining the endothelium of this zone of transition by various methods is described below.

\section{Material and Methods}

The eyes of the cat, rabbit, and rhesus monkey were studied, together with some human eyes with different pathological conditions, and one human eye which was clinically normal but was enucleated because of an orbital tumour.

The basic method used was again the silver impregnation method of McGovern $(1955,1956)$. The results were compared with those obtained by the replica and pseudo-replica methods, the latter mostly with additional staining. Even in the apparently normal eye, the possibility of functional changes in the secretion of the cement substance as well as of the covering substance should be borne in mind. The specimens used were either frozen sections or teased preparations; most of them were mounted in Apáthy's syrup to prevent excessive dehydration and shrinkage of the cement substance; some specimens mounted in Canada balsam showed that the cement substance seems much more resistant to dehydration after impregnation with silver.

\section{Findings}

The cells of the corneal endothelium, as they approach the corneal margin, become elongated in the meridional direction and sometimes form fanshaped groups. The shape and size of the cells as well as their outlines alter, and the endothelium shows a characteristic mosaic pattern. The cement substance often increases in thickness and sometimes covers the whole surface

* Received for publication September 30, 1957. 
of the cell with a thin argyrophil layer. A sudden change occurs as the endothelium passes the border of Descemet's membrane; the cells become much larger and flatter, and their outlines become sinuous. In shape they resemble the endothelial lining of lymphatic vessels, as they have been so beautifully depicted by Jancsó. As it is advantageous to remove the iris and ciliary body before the silver impregnation, and since some superficial lamellae of the trabeculae are easily removed thereby, it is difficult to obtain satisfactory specimens of the zone of transition without losing the superficial endothelial layer. The following procedure therefore proved to be better. After the eye had been dissected into its anterior and posterior halves, the lens was taken from the anterior segment and the whole anterior segment was impregnated by McGovern's method. This impregnated anterior segment was then divided sagittally and each half was cut on the freezing microtome in the frontal direction. Large, flat, intact endothelial cells are often to be found in such specimens; their surface mostly bears a thin film of a slightly argyrophil granular substance. The cement substance usually shows irregular thickening and often extends in a thin layer over the surface of the cells. (Fig. 1, and Fig. 2 opposite).

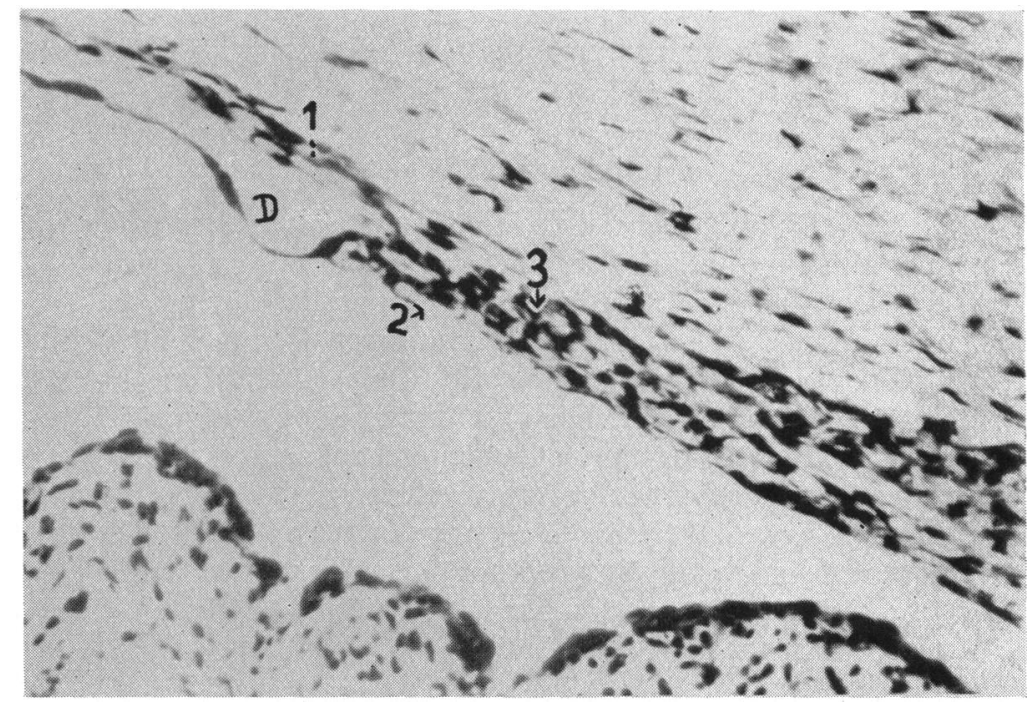

Fig. 1.-Sagittal section of anterior root of trabecular meshwork in the human eye, showing:

(D) Border of Descemet's membrane;

(1) Layers of anterior root of trabecula anterior to Descemet's membrane;

(2) and (3) Superficial and deeper layers of zone of transition.

Celodal embedding, impregnation by the Gros-Schulze method. $\times 366$.

The endothelial layer passes more equatorially into the trabecular endothelium; its cells become elongated in various directions, and their outlines become irregular and even invisible. Simultaneously, a thin layer of granular amorphous substance (which does not take up silver or only does 


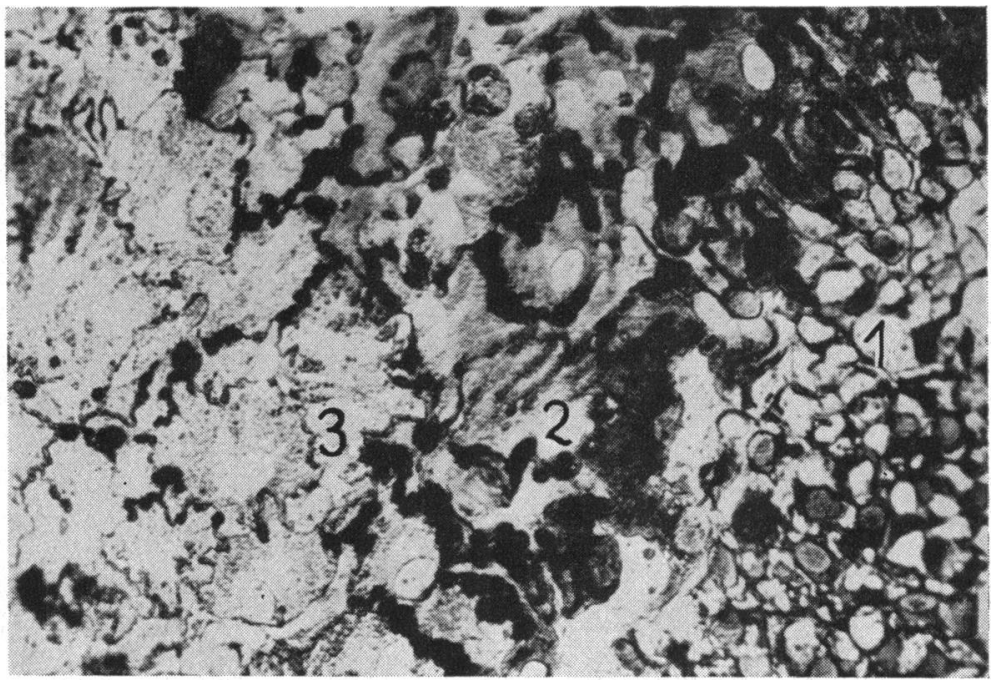

Fig. 2.-Endothelium of zone of transition in the human eye (zone 2 of Fig. 1), showing:

(1) Mosaic pattern of peripheral corneal endothelium;

(2) Increased covering substance, enlarged cell boundaries, and argyrophil ridges at beginning of zone of transition;

(3) Clearly outlined, large, flat endothelial cells of zone of transition. The covering substance seems to have been removed in the dissection.

Flat-frozen section, after impregnation by McGovern's method. $\quad \times 366$.

so irregularly) appears on the surface of the trabecular meshwork. This layer conceals most of the details of the trabecular meshwork and is perhaps the cause of the poor results obtained with the impregnation method on the trabecular endothelium itself. The secretion of the covering substance is apparently similar to that of the corneal endothelium and is apt to spread over the whole cellular surface. Functional changes in this secretion would require further study; the present description refers to the superficial cellular layer covering the inner surface of the zone of transition in the region of the anterior border of Schwalbe's ring. By focusing the microscope somewhat below the margin of Descemet's membrane in the direction of the corneal stroma, other layers of nearly regular endothelial lining are seen to cover the few (three or four) sheaths which represent the anterior roots of the trabecular meshwork. There the trabecula is fixed into the corneal stroma anterior to Descemet's membrane. In meridional sections some rows of nuclei appear which are more numerous than in the neighbouring stroma. In flat-frozen sections, and even more frequently in teased preparations, it is possible to see islands of this endothelial layer with a regular endothelial lining surrounded by irregular scattered keratoblasts of the corneal stroma (Fig. 3, overleaf).

This is an interesting feature of the anterior roots of the trabecular meshwork. Consequently, the flat sheaths are covered by a regular and continuous endothelial layer. The cell outlines are very conspicuous and sometimes- 


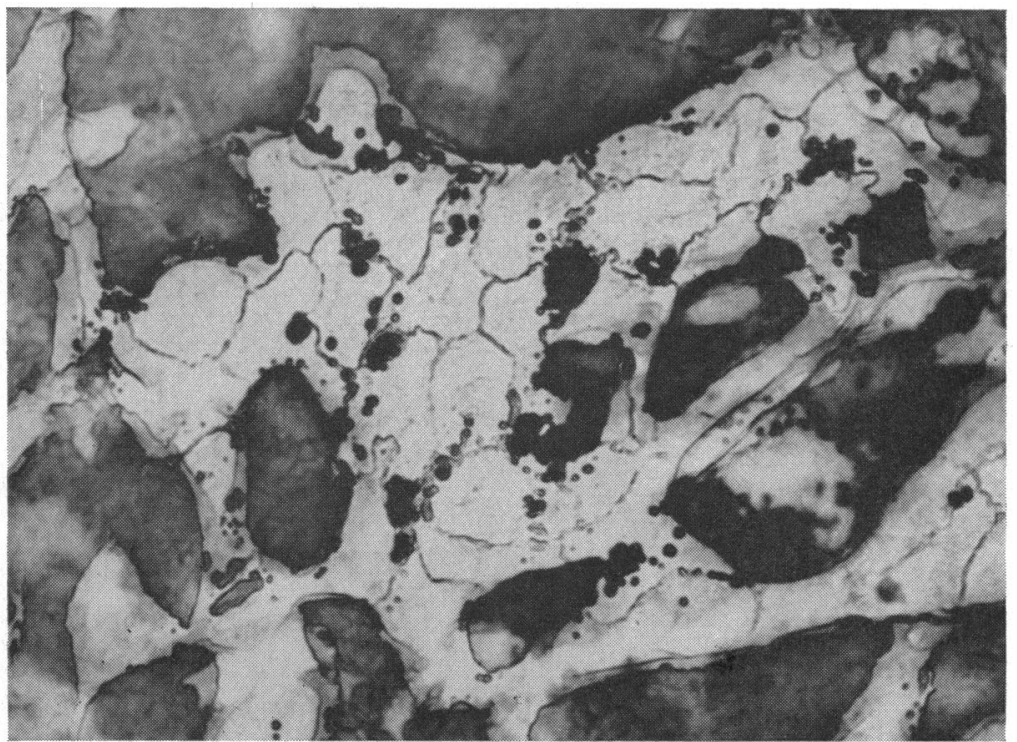

FIG. 3.-Anterior root of trabecula (Zone 1 of Fig. 1), showing islands of continuous layers of endothelium, cell outlines, and drop-like secretions of cementsubstance. Flat-frozen section impregnated by McGovern's method. $\times 366$.

especially near the border of Descemet's membrane-they appear to be irregularly thickened by the copiously secreted cement substance (Fig. 4)

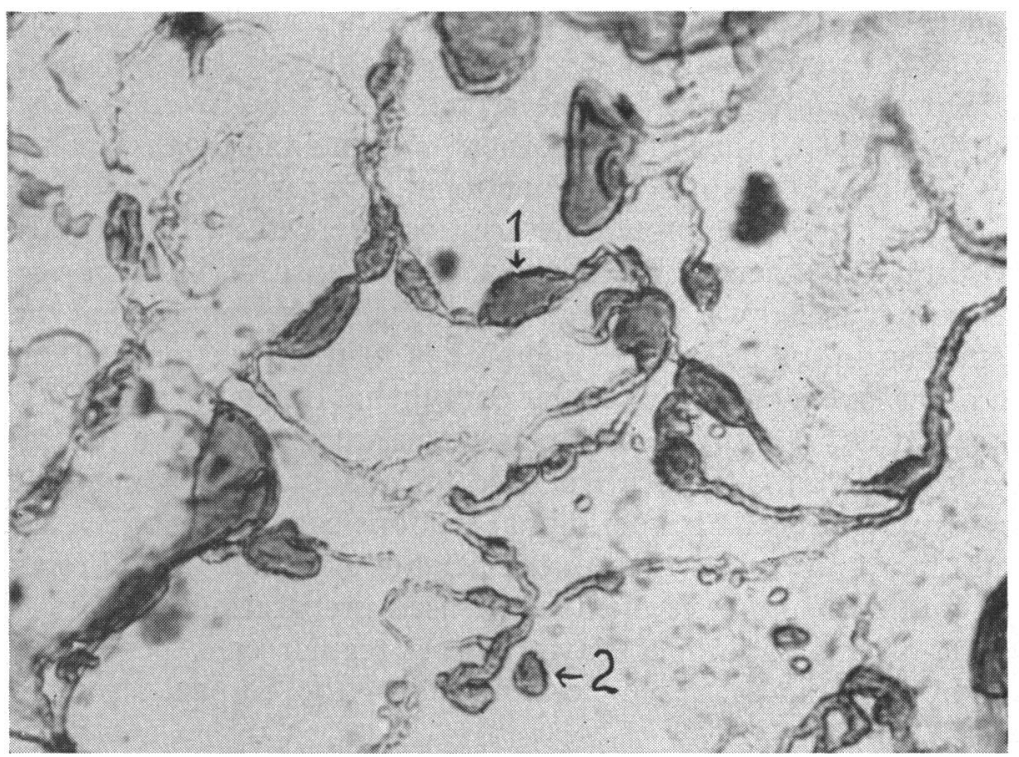

FIg. 4.-Deeper layers of zone of transition in the human eye (Zone 3 in Fig. 1):

(1) Large irregular fields with unevenly thickened cell outlines, and irregular secretion of cement substance

(2) Drop-like secretions of cement substance.

No cells are to be seen in this specimen. McGovern's method. $\quad \times 666$ 
More posteriorly, approaching the trabecular meshwork itself, the endothelial elements undergo changes similar to those described for the superficial layer in the same region. The amount of the intercellular substance varies in a similar way; it frequently forms larger blocks apparently binding the sheaths of the anterior roots of the trabecular meshwork together into a compact structure analogous to the corneal stroma.

These observations support the findings of earlier workers that the anterior roots of the trabecular meshwork were built from solid sheaths. The islands of the continuous endothelial layer in the tangential frozen sections differ widely from the adjacent corneal stroma, in which keratoblasts are bound in a loose cellular network, large hyaline blocks of cement substance isolating single elements of the cell structure. The irregularity of the more posteriorly situated elements in the zone of transition is caused by the appearance of pores in the loose trabeculae. The appearances are so irregular that some doubts may arise regarding their cellular origin, but in the more heavily impregnated specimens some nuclei could be traced.

Phase-contrast microscopy demonstrated beautiful pictures of endothelial cells with their nuclei and Golgi meshes (Fig. 5). On the surface of such cells were seen drops of granular argyrophil substance, evidently identical with the cement substance. In the specimens stained with toluidine blue, this cement substance stained only faintly. The dilated cell outlines were connected in some places by large blocks of cement substance.

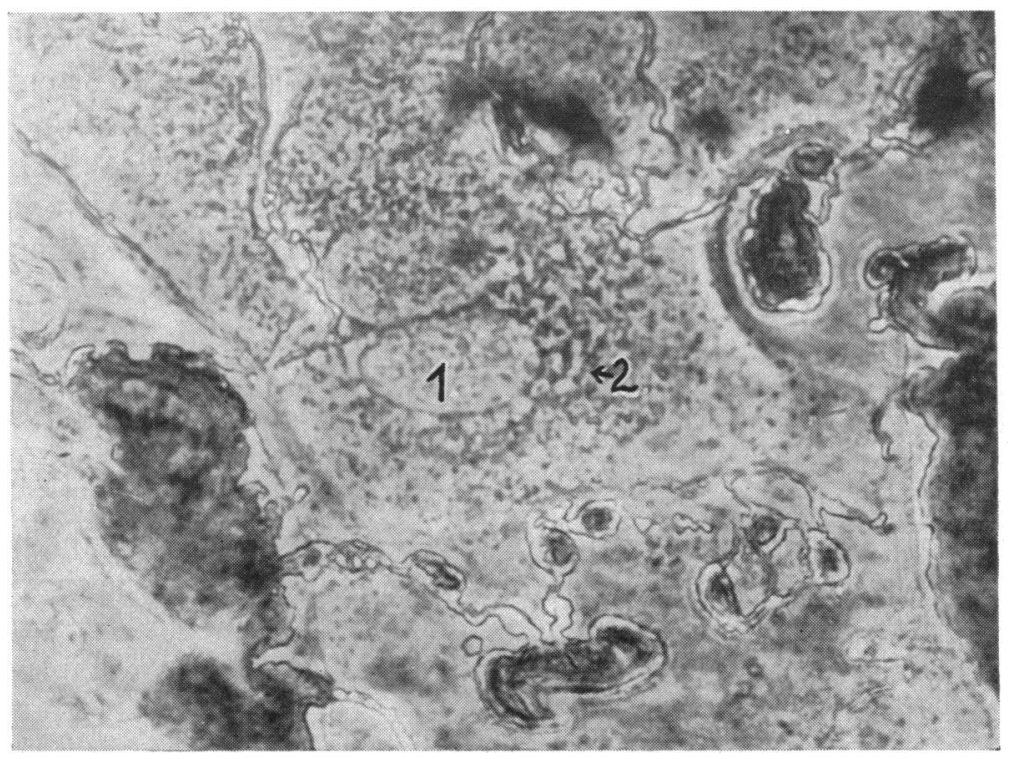

FIG. 5.-Same region of same specimen as in Fig. 3 photographed by the phasecontrast method, showing irregular outlines of one large field and drop-like secretions of cement substance. Within the outlines of a field is seen an endothelial element in the process of amitotic division. Nuclei (1) and Golgi meshes (2) are conspicuous.

The division of the cytoplasm is incomplete. McGovern's method. $\quad \times 1,666$. 


\section{Comment}

The fan-shaped groups of endothelial elements at the periphery of the cornea were first noticed during studies on the relief of the inner surface of the structures surrounding the anterior chamber. As Descemet's membrane arises at a later stage of embryonic development than the trabecular meshwork and ciliary muscle, it is tempting to consider these groups in relation to the anterior anchorage of the ciliary muscle tendons (Kusel, 1906; Rohen, 1956). The elongation of the endothelial nuclei reported by RochonDuvigneaud $(1892,1893)$ in the periphery of the cornea seems to be related to the deformation of the whole cell-body.

The sudden increase in size of the endothelial elements at the edge of Descemet's membrane seems to arise from the flattening which was observed by early workers (Rochon-Duvigneaud, 1892, 1893; Kolmer, 1936) to be present in this region. In studying the endothelial covering of the anterior surface of the iris (Vrabec, 1952), I noted that the endothelial elements which pass from the cornea to the iris around the lateral wall of the anterior chamber at an early stage of embryonic development are distinctly thinner than the comparatively small corneal elements.

Previous authors who have studied the minute anatomy of the trabecular meshwork have thought that cell outlines could be demonstrated in the endothelial covering. In the superficial layer of the endothelium in the region of the anterior border of Schwalbe's ring as well as in the deeper three or four layers of the anterior roots of the trabeculae anterior to Descemet's membrane, however, the endothelial elements still preserve their distinct boundaries and form continuous layers. In the early stages of embryonic development, these elements form a compact wedge of uniform tissue in direct continuation of the corneal endothelium. Further differentiation takes place only in the posterior part of the trabecular meshwork, where mechanical and functional needs lead to the formation of a spongy tissue. The process seems to be linked to that observed in my study of evolution of the superficial layer of the iris. It corresponds to the involution of mesenchymal elements which takes place in the lateral wall of the anterior chamber during the second half of intra-uterine life. On the other hand, the persistence of continuous endothelial layers in the anterior roots of the trabecular meshwork also appears to be of functional importance. As the aqueous humour passes from the anterior chamber through the filter of the posterior trabeculae to Schlemm's canal, the spaces in the trabecular meshwork may enlarge, allowing the aqueous humour to reach the corneal stroma along the sheaths of the anterior roots of the trabeculae. It seems probable that, by secreting the cement substance, the endothelial cells actively control this aqueous flow, which would otherwise cause a swelling of the corneal stroma. Clemens (1955) mentions the importance of hyaluronic acid and its esters as well as of hyaluronidase in controlling the water-balance of the tissues. This finding appears to be related to the accumulation of an amorphous sub- 
stance previously described (Vrabec, 1957), as well as to the deeper staining properties found in this part of the trabecular meshwork by Persson (1954).

\section{Summary}

The endothelial covering of the zone of transition in the region of the anterior border of Schwalbe's ring is described. Certain new aspects are considered from the morphological and physiological points of view. The peculiar fan-shaped groups of endothelial cells at the periphery of the cornea are closely related to the anterior anchorage of the ciliary muscle tendons. The endothelial elements immediately posterior to the margin of Descemet's membrane are conspicuously enlarged. The fact that the outlines of the cells are preserved in the endothelium of the zone of transition (in the superficial as well as in the deeper layers) may have some bearing on the control of the flow of aqueous humour to the corneal stroma through the sheaths of the trabecular meshwork.

\section{REFERENCES}

BÁRÁNY, E. (1954). Acta Soc. Med. upsalien., 59, 260.

ClEMENS, H. J. (1955). Acta histochem. (Jena), 1, 287.

KöLMER, W. (1936). "Handbuch der mikroskopischen Anatomie des Menschen", ed. W. von Mollendorff, band 3, teil 2. Springer, Berlin.

KüSEL (1906). Klin. Mbl. Augenheilk., 44 (2), 80.

MCGOVERN, W. J. (1955). J. Path. Bact., 69, 283. (1956). Ibid., 71, 1.

Persson, B. (1954). Quoted by Bárány (1954).

ROCHON-DUVIGNEAUD (1892). Arch. Ophtal., 12, 732. (1893). Ibid., 13, 20, 108.

ROHEN, J. (1956). Ophthalmologica (Basel), 131, 51.

VRABEC, F. (1952). Ibid., 123, 20. (1957). Brit. J. Ophthal., 41, 20.

(1958a). Ibid., 42, 529.

(1958b). Amer.J. Ophthal., (in press). 